\section{\$13. Effects of Roughness and Temperature on Low-energy Hydrogen Positive and Negative Ion Reflection from Silicon and Carbon Surfaces}

\author{
Yamaoka, H. (RIKEN SPring-8 Center), \\ Tanaka, N. (Osaka Univ.), \\ Nishiura, M. (Univ. Tokyo), \\ Tsumori, K., Kisaki, M., \\ Kato, S., Miyamoto, T. (Doshisha Univ.), \\ Kenmotsu, T. (Doshisha Univ.), \\ Wada, M., Sasao, M. (Doshisha Univ.), \\ Matsumoto, Y. (Tokushima Bunri Univ.)
}

Hydrogen ion-solid surface interaction leads to formation of both positive and negative ions at the surface, and is of particular importance in wall conditioning of plasma confinement devices. We have studied the fundamental processes of beam-surface interactions by measuring the positive and negative ions scattered from the solid surface by injecting low-energy (13 keV) light ion beams. ${ }^{1)}$ Both positive and negative ions from molybdenum, tungsten, vanadium alloy, and carbon nano wall were observed. The intensities of negative ions formed on these samples were the same order of magnitude. The negative ion yields from such high work function surfaces of graphite, $\mathrm{Si}$, and diamond are too large to be explained by conventional surface charge transfer models. We also found that the surface structure affected the reflection property. The results suggest that the surface structure is an important factor determining the intensities of scattered positive and negative ions. On the other hand, most experiments of the beam-surface interaction have been performed at room temperature. Here we report the surface roughness effect on the reflection properties at $\mathrm{Si}$ targets for a $1 \mathrm{keV} \mathrm{H}^{+}$beam injection. Reflection from $\mathrm{Si}$ and carbon surfaces are measured for comparison. Temperature effects of these materials are also studied.

We prepared a Si crystal, a rough-finished Si (a few $\mu \mathrm{m}$ roughness), and a graphite (carbon plate) samples. The $\mathrm{H}^{+}$beam energy was kept at $1 \mathrm{keV}$ and beam current was 10-40 nA at the target throughout this series of the experiment. The reflected ions were measured by a magnetic momentum analyzer consists of a pair of magnetic coils and a multi-channel plate set on a turntable. This analyzer system enables energy and angle resolved measurements of reflected positive and negative ions with single scan.

Reflection angle dependence of the reflected ion energy and intensity at several incident angles were measured for Si crystal, rough-finish Si crystal, and carbon plate. ${ }^{2)}$ FE-SEM images of the rough-finish SI crystal and carbon plate are shown in upper panels in Fig. 1. We define the incident and reflection angles with respect to the sample surface. The reflected ion intensity in the

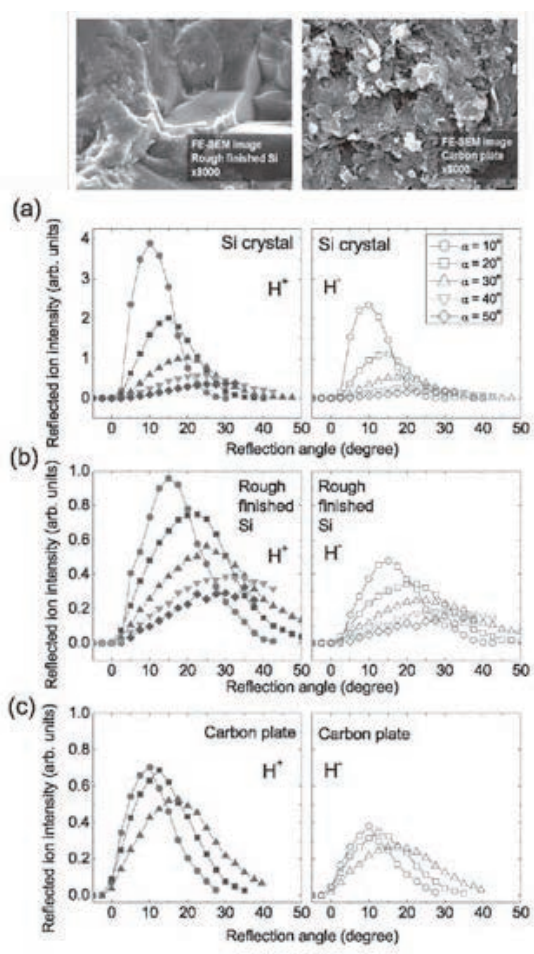

Fig. 1: Reflection angle $(\beta)$ dependence of the $\mathrm{H}^{+}$ and $\mathrm{H}^{-}$ion intensity reflected from (a) Si crystal, (b) rough-finish $\mathrm{Si}$, and (c) carbon plate. FE-SEM images for rough-finish $\mathrm{Si}$ and carbon plate the are also shown.

rough-finished $\mathrm{Si}$ was largely reduced compared to that in the Si crystal as shown in Figs. 1(a) and 1(b). The angular distribution of the rough-finished Si was slightly broader than that of Si crystal at $\alpha=10^{\circ}$ and that of the graphite was much broader than the others. Figure 1(c) shows result for the carbon plate (graphite). The graphite has a piled-up structure of small pieces horizontally. This result suggests that this structure has a similar effect on the reflected ion intensity angle distribution as the diffuse scattering in the rough-finished Si. Thus the structure and surface roughness affect on the scattering ion intensity and angle distribution particularly at lower incident angles. We also studied temperature dependence of the reflected ion intensity for these samples. Temperature-dependence of the $\mathrm{H}^{+}$ion intensity reflected from the Si crystal and graphite are measured. Both the Si crystal and rough-finished Si did not show clear temperature dependence, while reflected $\mathrm{H}^{+}$ion intensity decreases and $\mathrm{H}^{-}$ion intensity increases slightly as increasing the temperature of the graphite sample.

1) M. Wada et al., Plasma Device and Operations 17, $132(2009)$

2) N. Tanaka et al., Rev. Sci. Instrum. 85, 02C311 (2014). 